\title{
Deaths at UK immigration detention centres are the result of avoidable systemic failures, report says
}

Medical Justice has clarified that 35 deaths in detention were analysed for their report, which is covered in this News story (BMJ 2016;354:i4073, doi:10.1136/bmj.i4073), not 38 because they excluded three deaths from their analysis. The online text of Medical Justice's report has been amended to reflect this. 